\title{
A design of lens antenna with high gain
}

\author{
Darong Gao ${ }^{1, *}$ \\ ${ }^{1}$ The Electrical Engineering College, Northwest Minzu University, Gansu 730030, China
}

\begin{abstract}
In this paper, A lens antenna with high gain is proposed. The antenna is composed of the microstrip antenna and the hemisphere dielectric lens, and the lens is loaded on the top. The polyethylene is used to fabricated the hemisphere dielectric lens. The antenna has dimensions of $47.58 \mathrm{~mm} \times 47.58 \mathrm{~mm} \times 24.79 \mathrm{~mm}$, which is corresponding to the electrical size of $1.586 \lambda 0 \times 1.586 \lambda 0 \times 0.826 \lambda 0$, where $\lambda 0$ is the free-space wavelength at $10 \mathrm{GHz}$. The impedance bandwidth (return loss $<-10 \mathrm{~dB}$ ) is $12.7 \%(9.24 \mathrm{GHz}-10.51 \mathrm{GHz})$, and the peak gain is $9.06 \mathrm{dBi}$. The hemisphere dielectric lens can improve the gain of the microstrip antenna. The proposed lens antenna is suitable for wireless communications systems.
\end{abstract}

\section{Introduction}

In the recent years, with the development of technology and improvement of the antenna performance requirements, lens antenna becomes more and more attractive to the majority of researchers. In [1], The circular polarization plane luneberg lens antenna for wireless communication of millimeter wave was proposed, the lens antenna is fed by a microstrip antenna. A luneberg lens multiple-beam scanning antenna for millimeter-wave frequencies was introduced in [2], and the luneberg lens is filled by air, the antenna is fed by a planar antipodal linearly-tapered slot antenna. In [3], the beamspace large-scale multiple input multiple output lens antenna array for wireless power transfer was proposed. A two dimensional beam scanning K-band focal-shifted lens antenna was introduced in [4], the lens antenna is fabricated by three dimensional printing, the antenna features twodimensional beamscanability, high gain, and good matching. In [5], a fresnel zone plate lens antenna was proposed, the antenna is fed by the high gain patch antenna, and the operating frequency of the lens antenna is $60 \mathrm{GHz}$. In [6], the high production tolerance and small antenna substrate antenna for $77 \mathrm{GHz}$ long-range radar is proposed, the antenna composed of the dielectric lens and the pyramidal horn.

The lens antennas have been widely used in millimeter-wave frequencies, however, the low frequency lens antenna has rarely been reported.

In this paper, a high gain lens antenna is reported. The lens antenna is composed of the microstrip antenna and hemisphere dielectric lens, and the microstrip antenna is fit closely together. The impedance bandwidth (return loss $<-10 \mathrm{~dB})$ is $12.7 \%(9.24 \mathrm{GHz}-10.51 \mathrm{GHz})$, and the peak gain is $9.06 \mathrm{dBi}$.

\footnotetext{
*Corresponding author: 568629455@qq.com
} 
This paper is organized as follows. The Section 2 introduces the design method of antenna. The simulated results of the antenna are explained in section 3 . The role of key parameters is discussed in section 4 , and a brief conclusion is given in section 5 .

\section{Antenna design}

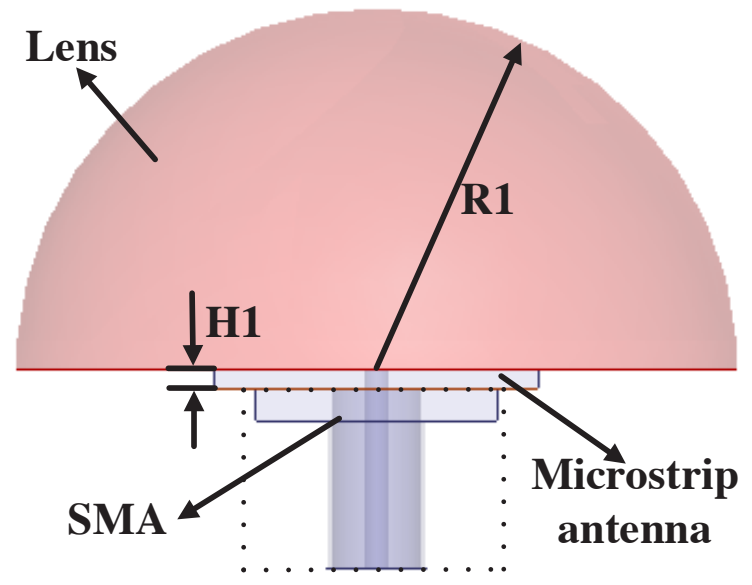

(a)

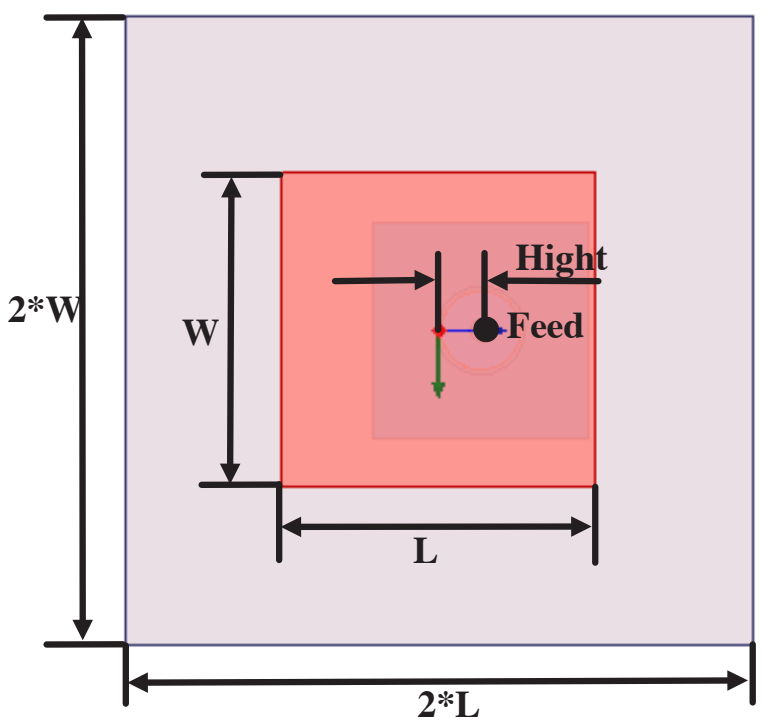

(b)

Fig. 1. Geometry of the proposed antenna. (a) side view. (b) geometry of the microstrip antenna.

In this section, the lens antenna design process is devoted. The geometry and sizes of the lens antenna are shown in Fig. 1 and Table. 1, respectively.

The microstrip antenna is the feed source, and fed by $50 \Omega$ SMA. The microstrip antenna was constructed on the substrate ROGERS 5880, the dielectric constant is 2.2 , the thickness is $1 \mathrm{~mm}$ and the loss tangent is 0.0009 . 
Table 1. Units for magnetic properties.

\begin{tabular}{|c|c|}
\hline Margin & $\mathbf{m m}$ \\
\hline R1 & 23.79 \\
\hline H1 & 1.0 \\
\hline L & 8.65 \\
\hline W & 9.05 \\
\hline Hight & 3.27 \\
\hline
\end{tabular}

The radius of the hemisphere dielectric lens is $23.79 \mathrm{~mm}$, and the polyethylene is used, the dielectric constant is 2.55 , and the tangent is 0.0011 .

The simulated results for the microstrip antenna and the lens antenna, The peak gain of the microstrip antenna is $7.8 \mathrm{dBi}$. After loading the hemisphere dielectric lens, the peak gain is $9.06 \mathrm{dBi}$, respectively. The impedance bandwidth and the radiation characteristic are improved by the hemisphere dielectric lens.

\section{Antenna simulated results}

The simulated results of the proposed antenna are shown in Fig. 2 and Fig. 3. It clear that the impedance bandwidth is $12.7 \%(9.24 \mathrm{GHz}-10.51 \mathrm{GHz})$, the peak gain is $9.06 \mathrm{dBi}$.

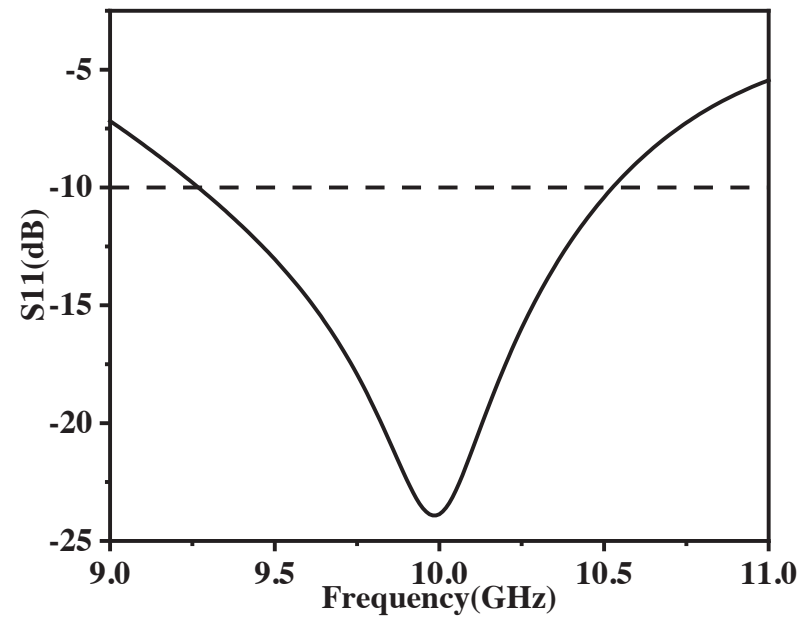

Fig. 2. Simulated reflection coefficient.

\section{Parametric study}

The parameters are studied and the key parameters are determined, i. e. the radius of the lens, the length and the width of the microstrip antenna.

Fig. 4 shows the effect of the microstrip antenna length $L$ on the bandwidth of the proposed antenna. It is clear that the resonance point is increased with the length decreased. Other parameters have little influence on the antenna performance. 


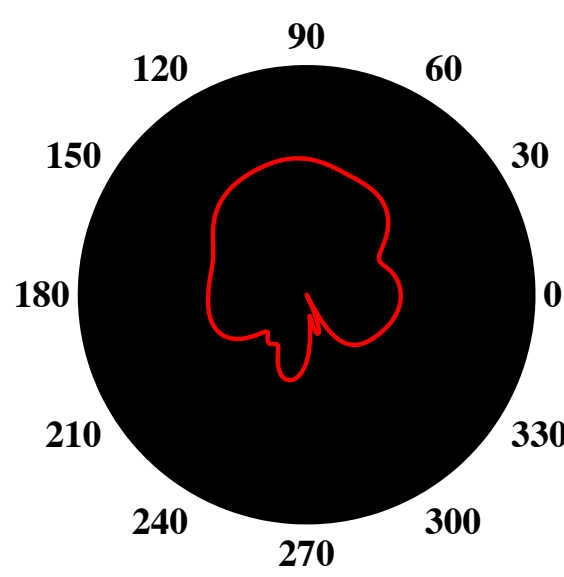

Simulated copolar

(a)

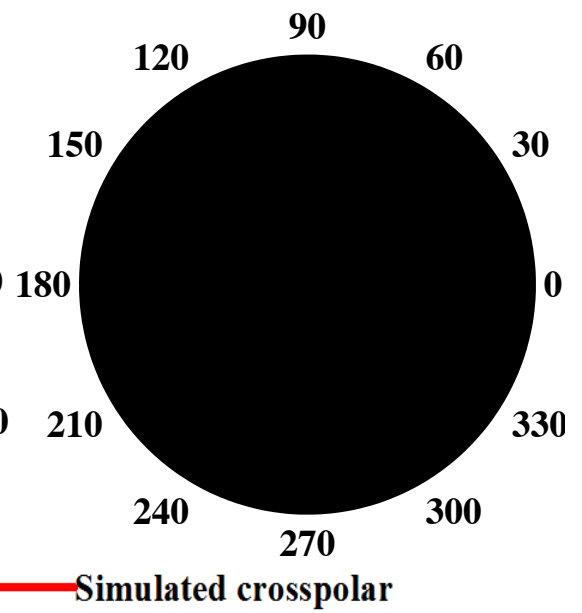

(b)

Fig. 3. Geometry of the proposed antenna. (a) side view. (b) geometry of the microstrip antenna.

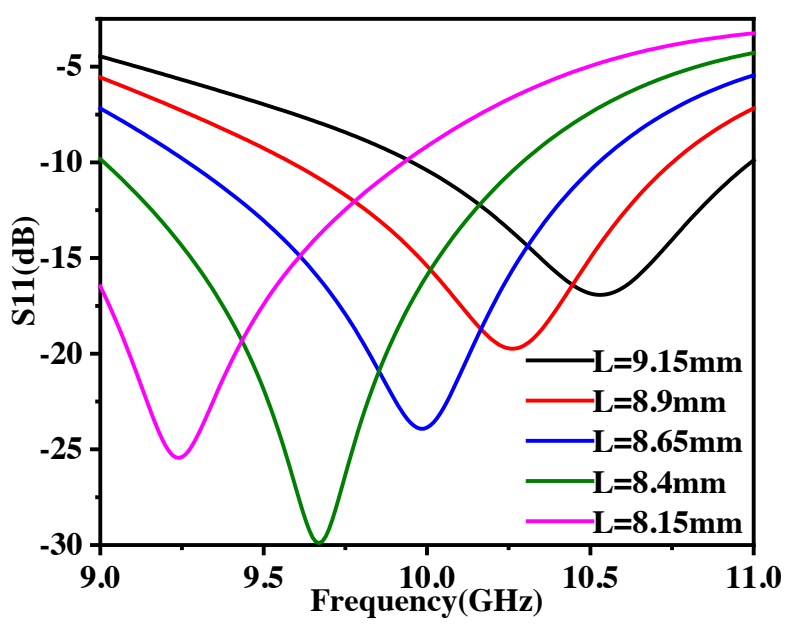

Fig. 4. Photograph of reflection coefficient for different length of the microstrip antenna.

\section{Conclusion}

Feasibility of a lens is demonstrated for high gain. The antenna is composed of a microstrip antenna and a hemisphere dielectric lens. The antenna exhibits a bandwidth of $12.7 \%$ from 9.24 GHz to $10.51 \mathrm{GHz}($ return loss $<-10 \mathrm{~dB}$ ), and the peak gain is $9.06 \mathrm{dBi}$. The antenna has dimensions of $47.58 \mathrm{~mm} \times 47.58 \mathrm{~mm} \times 24.79 \mathrm{~mm}$, the equivalent to $1.586 \lambda 0 \times 1.586 \lambda 0 \times 0.826 \lambda 0$, where $\lambda 0$ is the free-space wavelength at the resonance frequency of $10 \mathrm{GHz}$. The hemisphere dielectric lens observably improved the gain of the microstrip antenna. The antenna has a wide range of wireless application foreground communication system.

This work is partially supported by the Fundamental Research Funds for the Central Universities(31920190153). 


\section{References}

1. Z. Shi, S. Yang, S. W. Qu, et al, "Circularly polarised planar Luneberg lens antenna for mm-wave wireless communication.” Electronics Letters, vol. 52, no. 15, pp. 12811282, July 2016.

2. C. Z. Hua, X. D. Wu, N. Yang, and W. Wu, “Air-Filled Parallel-Plate Cylindrical Modified Luneberg Lens Antenna for Multiple-Beam Scanning at Millimeter-Wave Frequencies." IEEE Transactions on Microwave Theory and Techniques, vol. 61, no. 1, pp. 436-443, Jan 2013.

3. S. Zhong. and X. D. Wang, “Wireless Power Transfer by Beamspace Large-Scale MIMO With Lens Antenna Array.” IEEE Transactions on Wireless Communications, vol. 18, no. 2, pp. 1390-1403, Feb 2019.

4. B. Zhang, K. M. Huang, "A Hybrid 3D Printed K-Band Focal-Shifted TwoDimensional Beam Scanning Lens Antenna with Non-Uniform Feed,” IEEE AsiaPacific Microwave Conference (APMC). IEEE, 2020.

5. P. L. Chi, C. H. Pao, M. H. Huang, et al. "High-Gain Patch-Fed 3D-Printing Fresnel Zone Plate Lens Antenna for 60-GHz Communications,” 2018 IEEE International Symposium on Antennas and Propagation \& USNC/URSI National Radio Science Meeting. IEEE, 2019.

6. Kuriyama A, Nagaishi H, Kuroda H. "Horn and lens antenna with a small antenna substrate and high production tolerance for $77 \mathrm{GHz}$ automotive long-range Radar," IEEJ Transactions on Electrical and Electronic Engineering, vol. 14, no. 3, 2019, 14. 